\title{
Remotely excited Raman optical activity using chiral plasmon propagation in Ag nanowires
}

\author{
Mengtao Sun ${ }^{1 *}$, Zhenglong Zhang ${ }^{1 *}$, Peijie Wang ${ }^{2}$, Qiang $\mathrm{Li}^{1}$, Fengcai Ma ${ }^{3}$ and Hongxing Xu ${ }^{1}$
}

We experimentally investigated remotely excited Raman optical activity (ROA) using propagating surface plasmons in chiral Ag nanowires. Using chiral fmoc-glycyl-glycine-OH (FGGO) molecules, we first studied the local surface plasmon-enhanced ROA. We found that the Raman intensity can be excited by left- and right-circularly polarized lights and that the circular intensity difference (CID) can be significantly enhanced. Second, by selecting vibrational modes with large Raman and ROA intensities that are not influenced by chemical enhancements, we studied remotely excited ROA imaging and the CID of FGGO molecules by propagating a plasmonic waveguide using Ag chiral nanostructures. When laser light was radiated on one of the Ag terminals, the measured CID of the FGG at the other terminal showed little change compared to the local excited CID. Meanwhile, when the laser light was radiated on the Ag nanowires (not on the terminals) and was coupled to the nearby nanoantenna, the CID of the ROA could be manipulated by altering the coupling angle between the Ag nanowires. To directly demonstrate the propagation of ROA along the nanowire and its remote detection, we also measured the remotely excited ROA spectra. Our experimental method has the potential to remotely determine the chirality of molecular structures and the absolute configuration or conformation of a chiral live cell.

Light: Science \& Applications (2013) 2, e112; doi:10.1038/lsa.2013.68; published online 22 November 2013

Keywords: Ag nanowires; chiral plasmons; plasmon propagation; Raman optical activity; remote excitation

\section{INTRODUCTION}

Raman optical activity (ROA) ${ }^{1-3}$ results in differences in the Raman spectra excited by right- and left-circularly polarized light; this activity is extremely sensitive to the chirality of the molecular structure ${ }^{4-6}$ and can reveal the absolute molecular configuration ${ }^{5}$ or conformation. ${ }^{6}$ Several excellent reviews of ROA have been reported. ${ }^{7-9}$ The dimensionless circular intensity difference (CID) introduced by Barron and Buckingham ${ }^{1}$ provides an experimental observable of this phenomenon:

$$
\Delta=\frac{\left|I_{L}-I_{R}\right|}{\left|I_{L}+I_{R}\right|}
$$

where $I_{R}$ and $I_{L}$ are the Raman scattering intensities of right- and leftcircularly polarized light, respectively. Although chiral-sensitive vibrational spectroscopy has become increasingly popular in biological applications, this approach has an obvious flaw that is difficult to overcome: the ROA scattering intensities are $10^{-3}-10^{-5}$ times the intensities of the parent Raman scattering. Due to the low intensity of ROA, this technique has certain limitations and requires higher sample concentrations and longer data collection periods.

The intrinsic weakness of ROA can be resolved by utilizing surface plasmon enhancement. ${ }^{10-17}$ The local electric field and field gradients generated by plasmon resonance can significantly enhance the ROA and CID. ${ }^{9}$ Surface-enhanced ROA is attributed to the coupling between the electric dipole and the electric quadrupole terms in the process of Raman scattering. ${ }^{16}$ The quadrupole transitions can only be significantly excited in an oriented molecule close to the metallic substrate; under far-field excitations, the transitions are weak.

In general, the influence of chemical enhancement is difficult to distinguish in surface-enhanced ROA. Some vibrational modes are strongly influenced by chemical enhancements, while for other modes, chemical enhancements can be ruled out based on the characterization of selective chemical enhancement. Thus, we must carefully select vibrational modes that exhibit both strong Raman and CID intensities for which there is no influence of chemical enhancement.

The propagation of a surface plasmon polariton along metal nanowires enables the incident light to transfer through a subdiffractionlimit nanowire over micrometer-scale distances. ${ }^{18}$ This unique property of surface plasmons enables the achievement of miniaturized photonic nanowires and high-resolution spectroscopy and microscopy. Remotely excited surface-enhanced Raman scattering (SERS) produced by means of a propagating surface plasmon in Ag or Au nanowires has been experimentally studied. ${ }^{19-21}$ Compared to local SERS techniques that excite targets directly, the remote excitation method can avoid specimen damage caused by the laser, which may be critical in some cases, for example, in intercellular chiral live cells.

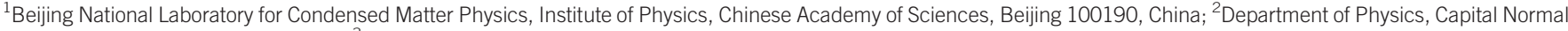
University, Beijing 100048, China and ${ }^{3}$ Department of Physics, Liaoning University, Shenyang 110036 , China

*These authors contributed equally to this work.

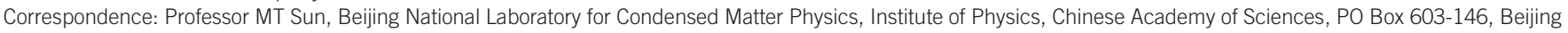
100190, China 
Further, chiral plasmonic nanostructures ${ }^{22}$ can be used to manipulate and enhance the CID of intrinsic chiral molecules. Thus, it is desirable to design plasmonic nanostructures for local and remote chiral sensing. ${ }^{23}$

In this communication, we report an experimental realization of remotely excited ROA via chiral plasmons propagating on Ag nanowires. To directly demonstrate the propagation of ROA along a nanowire and its remote detection, we also measured the remotely excited ROA spectra. This approach has the potential to remotely determine the chirality of molecular structures and the absolute configuration or conformation of chiral living cells.

\section{MATERIALS AND METHODS}

Fmoc-glycyl-glycine-OH (FGGO) was obtained from Aldrich Chemical (China) Co. and was used as received. The Raman and ROA spectra (Figure 1) were collected using the backscattering SCP Biotools $\mu$-ChiralRAMAN-2X. The laser light had a wavelength of $532 \mathrm{~nm},{ }^{24}$ and a power of $300 \mathrm{~mW}$ was radiated on the sample. The total collection time was $4 \mathrm{~h}$.

Single crystalline Ag nanowires were synthesized using chemical methods. ${ }^{25}$ High-resolution scanning electron microscopy (SEM) images were acquired at a voltage of $10 \mathrm{kV}$. The $\mathrm{Ag}$ nanowires were mixed with FGGO ethanol solution $(0.04 \mathrm{M})$ for $24 \mathrm{~h}$ and were then dropped on a glass substrate. To examine the influence of the substrate on the shift in the Raman frequencies for SERS, the local SERS was measured with linearly polarized light excited at $632.8 \mathrm{~nm}$, using a Leica microscope installed with a confocal Raman spectroscopic instrument (Invia; Renishaw, UK) with a $100 \times$ objective and a numerical aperture of 0.85 . Then, local and remote Raman images of the molecular vibrational mode at $1593 \mathrm{~cm}^{-1}$ were obtained using rightand left-circularly polarized light, respectively. This vibrational mode exhibits large Raman ROA intensities and is not influenced by chemical enhancements. The optical images were recorded using a TE-cooled CCD detector installed on the microscope. The Raman images were remotely measured. Laser light at $632.8 \mathrm{~nm}$ (power of approximately
$7 \mathrm{~mW}$ ) was radiated on the crossing point (point a in Figure 2a) using left- and right-circularly polarized light, respectively, and the Raman signals were measured at the remote terminals of the nanowires (points b, $c$ and $d$ in Figure 2a). This method of measuring a single Raman mode propagating along a nanowire has been described in detail in Refs. 19, 20, 26 and 27; this approach is generally called Raman imaging in SERS. Finally, using Equation (1), local and remote surface plasmon-enhanced ROA imaging can be obtained. To directly demonstrate the propagation of the ROA along the nanowire and its remote detection, we also measured the remotely excited ROA spectra.

We fabricated a metal-insulator-semiconductor nanowire plasmonic waveguide system consisting of a wet-chemically grown $\mathrm{Ag}$ wire, which was then covered by $\mathrm{Al}_{2} \mathrm{O}_{3}$ films of varying thickness using an atomic layer deposition (ALD) system (Savannah-100; Cambridge NanoTech, USA) to achieve a well-defined thickness. Subsequently, the sample was spin-coated with highly luminescent CdSe/ZnS Organic Quantum Dots (Qdot 655; Invitrogen, China).

Theoretical simulations were performed using Gaussian 09. ${ }^{28}$ The geometry of FGGO at the ground state was optimized at the level of density functional theory, ${ }^{29}$ the B3LYP functional, ${ }^{30}$ and the $6-311+g(2 d, p)$ basis set. Using the optimized ground state geometry of FGGO, the Raman and ROA spectra of FGGO were then simulated.

\section{RESULTS AND DISCUSSION}

The experimental Raman and ROA spectra of FGGO are shown in Figure $1 \mathrm{a}$ and $1 \mathrm{~b}$. The highest intensities for both spectra appear at $1607 \mathrm{~cm}^{-1}$, with a CID value of $\Delta=0.57 \%$. The vibrational mode of this peak is shown in Figure 1c, which provides the best candidate for studying the remote excitation ROA using chiral plasmon propagation in $\mathrm{Ag}$ nanowires. For the vibration mode at $1607 \mathrm{~cm}^{-1}$, both the Raman and CID intensities are at their maxima, based on the experimental spectra shown in Figure 1. This vibrational mode corresponds to the symmetric vibration of ${ }^{1} \mathrm{H}$-fluorenyl, and there are no $\mathrm{O}$ or $\mathrm{N}$ atoms in this group. In general, $\mathrm{O}$ and $\mathrm{N}$ atoms directly interact with metal, and thus, vibrational modes for groups including these atoms
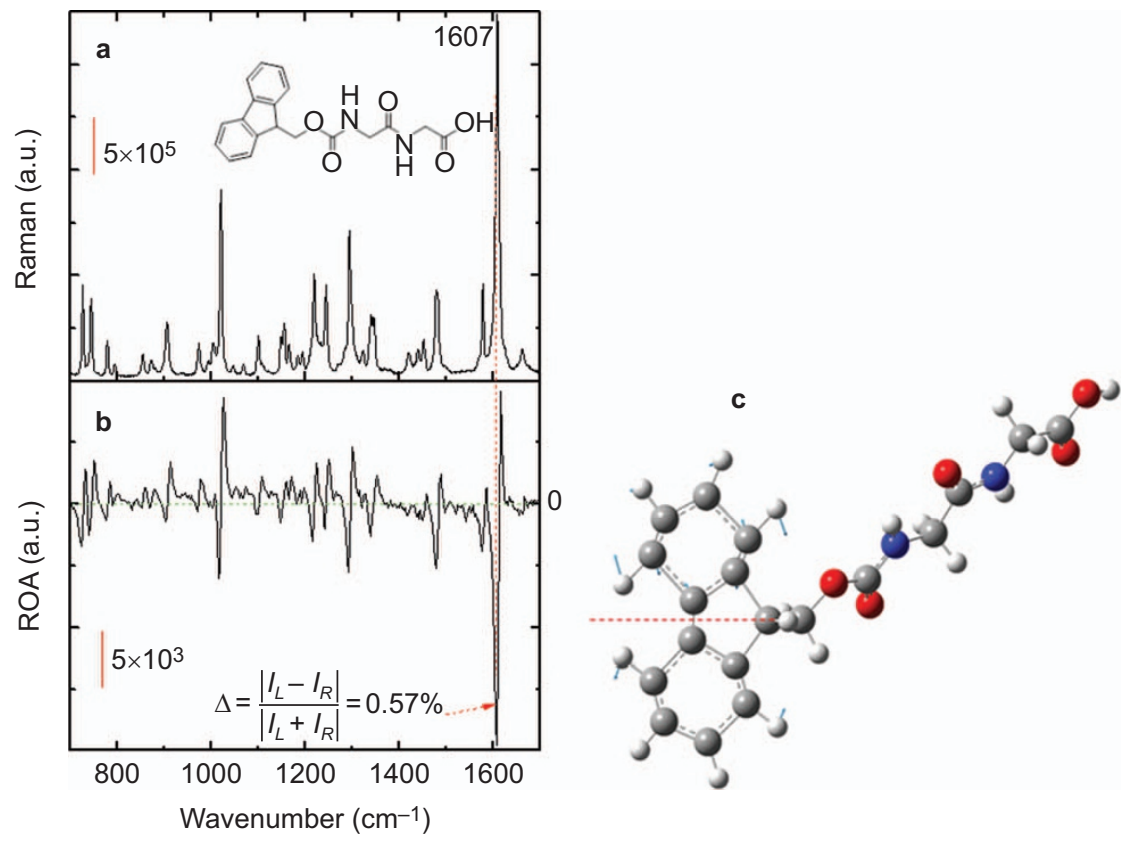

Figure 1 Experimental Raman and ROA spectra and the vibrational mode of FGGO at $1607 \mathrm{~cm}^{-1}$. (a) Raman spectrum. (b) ROA spectrum. (c) Vibrational mode of FGGO at $1607 \mathrm{~cm}^{-1}$. FGGO, fmoc-glycyl-glycine-OH; ROA, Raman optical activity. 

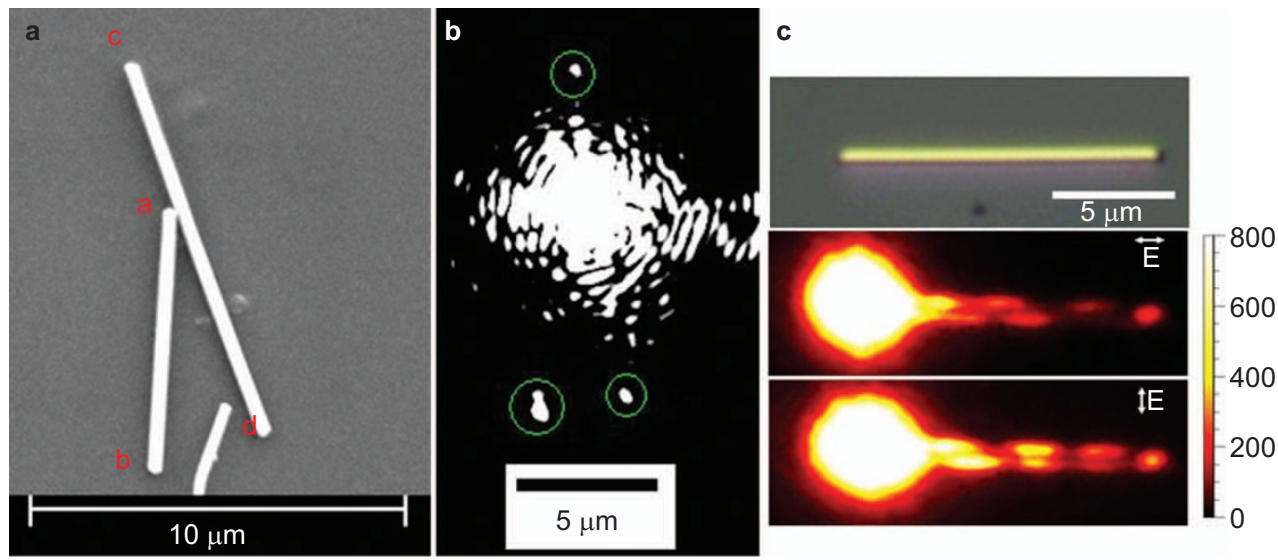

Figure 2 The structure of the Ag nanowires and the propagation of surface plasmons in the Ag nanowires. (a) Structure of the Ag nanowires. (b) Surface plasmons propagating in the Ag nanowires. (c) Optical image of a single nanowire and evidence of the chiral propagation of surface plasmons in the Ag nanowires. The chirality can be manipulated by the polarization of the laser.

are usually strongly influenced by chemical enhancements. In this case, there is no direct interaction between the group and the metal, and hence, there is no chemical enhancement for this vibrational mode. The assignments of the other vibrational modes with relatively strong Raman and CID intensities are given in Supplementary Fig. S1.

A glass substrate was used in the experiments. An SEM image of the Ag nanowires is shown in Figure 2a; the crossing angle between the nanowires was found to be approximately $20^{\circ}$. Figure $2 \mathrm{~b}$ demonstrates the propagation of plasmons in the Ag nanowires, and the light can be clearly observed at the terminals of the nanowires. To reveal the chiral propagation of the surface plasmons in the nanowire, the simplest nanostructure (a single nanowire) was used. Figure $2 \mathrm{c}$ shows an optical image of chiral surface plasmons propagating along a single nanowire. The degree of this type of chiral propagation can be manipulated via the polarization of the laser.
The SERS spectrum of locally excited FGGO at the crossing point of the Ag nanowires is shown in Supplementary Fig. S2 for linearly polarized light. The vibrational mode at $1607 \mathrm{~cm}^{-1}$ in Figure la was shifted to $1593 \mathrm{~cm}^{-1}$ due to the interaction between the FGGO and the Ag nanowires. Finally, local and remote Raman images of FGGO at $1593 \mathrm{~cm}^{-1}$, excited by left- and right-circularly polarized light, are shown in Figure $3 \mathrm{a}$ and $3 \mathrm{~b}$, respectively. As discussed above, this vibrational mode exhibits large Raman and ROA intensities, without the influence of chemical enhancement. Due to the chiral properties of the nanowire structure and the propagating surface plasmon, we observed different local and remote intensities of the Raman imaging at $1593 \mathrm{~cm}^{-1}$. The Raman intensities at these four points are significantly stronger when excited by the left circular polarization in comparison to the right circular polarization. Using Equation (1), the fitted CIDs at four points were obtained, as shown in Figure $3 \mathrm{c}$. The

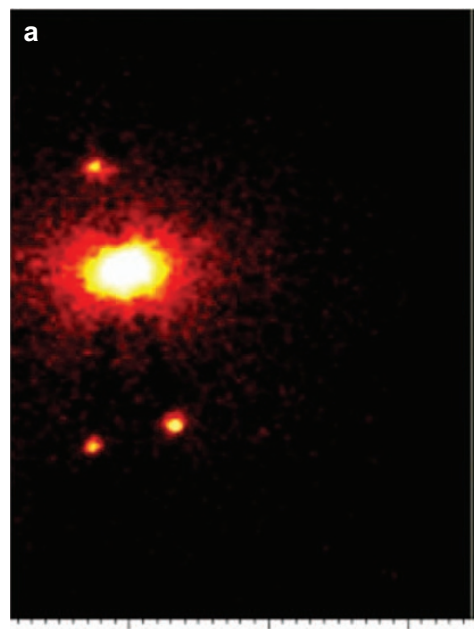

0 Microns ( $\mu \mathrm{m})$

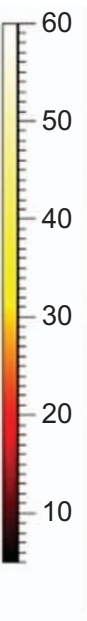

20

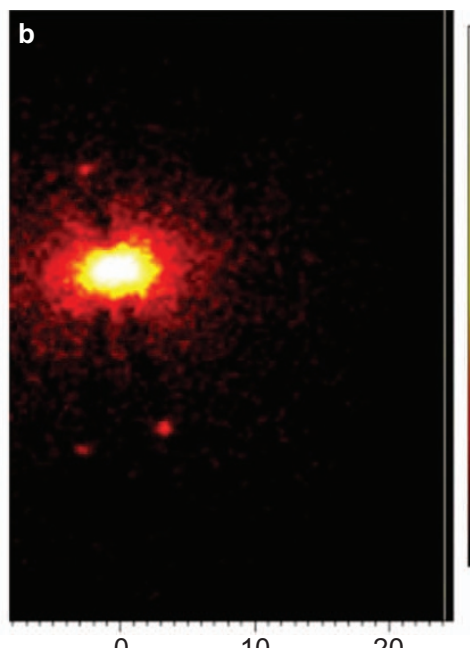

Microns $(\mu \mathrm{m})$
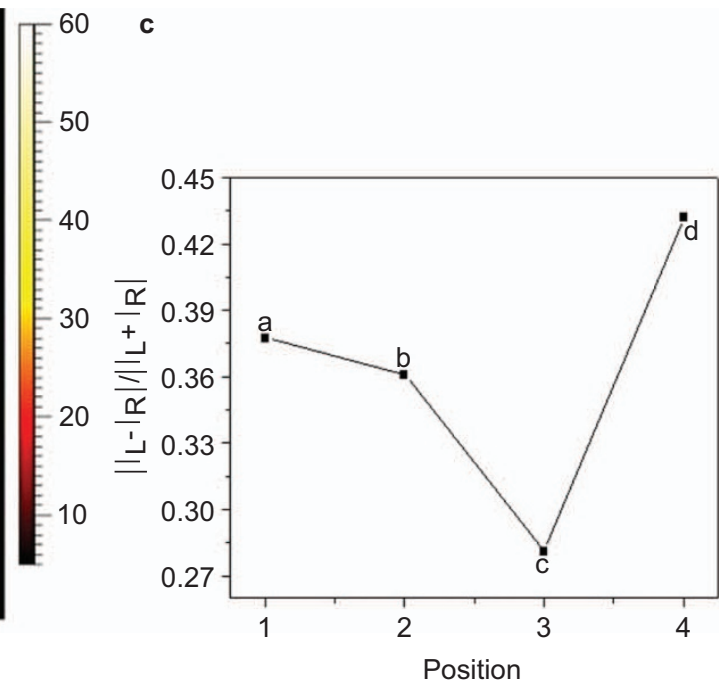

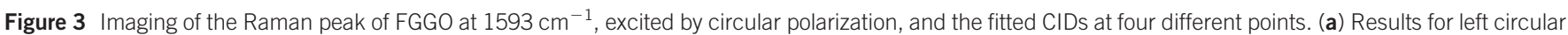

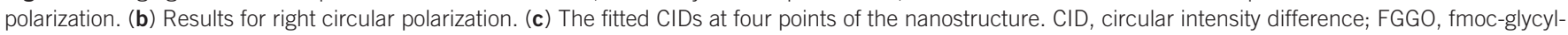
glycine- $\mathrm{OH}$. 

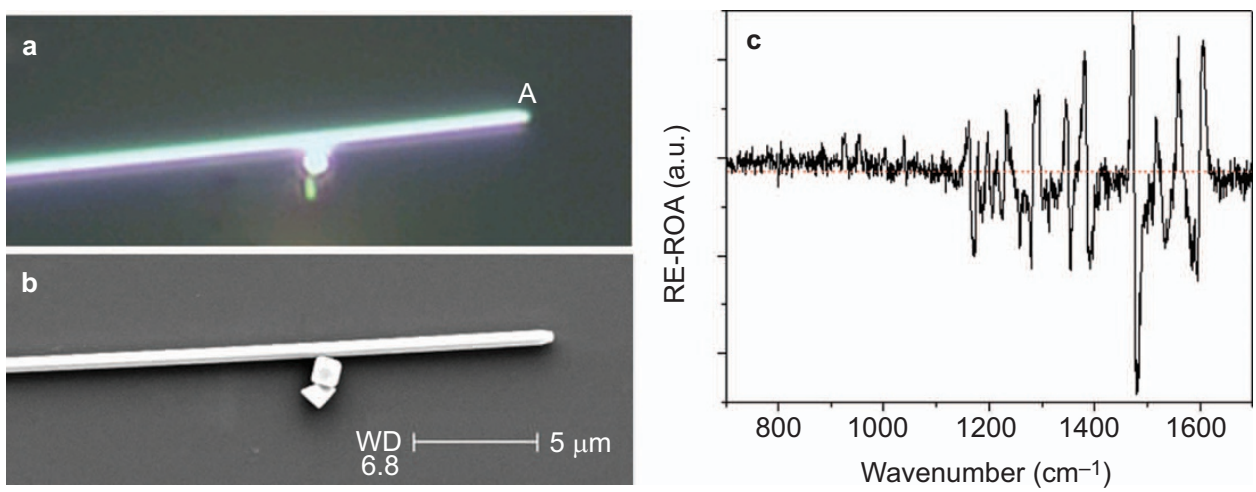

Figure 4 The nanostructure of the nanowire and the nanoparticle and the remotely excited ROA. (a) Optical imaging. (b) SEM imaging of the nanostructure of the nanowire and the nanoparticle. (c) The remotely excited ROA. ROA, Raman optical activity; SEM, scanning electron microscopy.

CIDs are significantly enhanced compared to the intrinsic molecular powder. Furthermore, the three CIDs at the three terminals of the Ag nanowires are also significantly different.

A comparison of the CID at crossing point A (the point at which the laser is radiated) to that at point $\mathrm{B}$ (the remote terminal of the same $\mathrm{Ag}$ nanowire) shows that the change in the CID is small. Therefore, the chiralities of both the propagating surface plasmon and the molecule do not change from one terminal to the other. In contrast, when the plasmon is coupled into the Ag nanowire at some point along the Ag nanowire close to the nanoantenna, the CIDs at the two remote terminals are very different and can be manipulated by the chiral nanostructure. In our case, the coupling angle between two Ag nanowires, the morphology of the Ag terminals, the distance of the coupling, etc., can be used to manipulate the chirality of the propagating surface plasmon. ${ }^{31,32}$ The changing chirality of the propagating surface plasmon can further change the molecular chirality and thus alter the CID value. In our fabricated nanostructure, when the crossing angle was less than $90^{\circ}$, the CID increased; when the crossing angle was larger than $90^{\circ}$, the CID decreased. Thus, we experimentally imaged remotely excited ROA using chiral plasmon propagation in Ag nanowires, and this method has the potential to remotely determine molecular chirality or the absolute configuration or conformation of a chiral living cell.

To directly demonstrate the propagation of ROA along the nanowire and its remote detection, we also measured the remotely excited ROA spectra (Figure 4). Figure $4 \mathrm{a}$ and $4 \mathrm{~b}$ show optical and SEM images of the Ag nanowire and a nanoparticle as the nanoantenna for the electromagnetic enhancement. The remotely excited Raman spectra were obtained using left and right polarized light (Supplementary Fig. S3). The laser was radiated on terminal A, and the signals were collected from the crossing point between the nanoparticle and the nanowire. Figure $4 \mathrm{c}$ displays the remotely excited ROA spectrum resulting from the propagating plasmonic waveguide, which is significantly different from the molecular ROA shown in Figure 1b. For some of the Raman peaks in Figure 4c, the signals were stronger than that at $1593 \mathrm{~cm}^{-1}$, which arose from chemical enhancements or the chiral nanostructures. It is known that chiral nanostructures can produce a chiral propagating plasmonic waveguide, which may also selectively enhance certain Raman peaks in the remotely excited ROA. A detailed analysis of the relationship among the ROA, the chemical mechanism and the chiral propagating plasmonic waveguide is currently being pursued.

\section{CONCLUSIONS}

We experimentally achieved remotely excited ROA imaging by selecting a single vibrational mode propagating along a nanowire, using a chiral plasmon propagating in Ag nanowires. The local and remote Raman intensity of FGGO at $1593 \mathrm{~cm}^{-1}$ were significantly enhanced when excited by left- and right-circularly polarized light. This vibrational mode exhibited large Raman and ROA intensities, without the influence of chemical enhancements. Furthermore, the local and remote CIDs, which were also significantly increased, were fitted and can be successfully manipulated by the nanostructure of the Ag nanowires. To directly demonstrate the propagation of ROA along the nanowire and its remote detection, we also measured the remotely excited ROA spectra. This work may be potentially utilized to remotely investigate the chirality of molecular structures, as well as the absolute configuration or conformation of chiral living cells.

\section{ACKNOWLEDGMENTS}

This work was supported by the National Natural Science Foundation of China (11374353 and 11274149) and the Program of Shenyang Key Laboratory of Optoelectronic Materials and Technology (F12-254-1-00).

1 Barron LD, Buckingham AD. Rayleigh and Raman scattering from optically active molecules. Mol Phys 1971; 20: 1111-1119.

2 Barron LD, Bogaard MP, Buckingham AD. Raman scattering of circularly polarized light by optically active molecules. J Am Chem Soc 1973; 95: 603-605.

3 Hug W, Kint S, Bailey GF, SchererJR. Raman circular intensity differential spectroscopy. Spectra of (2)-a-pinene and (1)-a-phenylethylamine. J Am Chem Soc 1975; 97: 5589-5590.

4 Sebestík J, Bour P. Raman optical activity of methyloxirane gas and liquid. J Phys Chem Lett 2011 ; 2: 498-502.

5 Hopmann KH, Sebestík J, Novotna J, Stensen W, Urbanova M et al. Determining the absolute configuration of two marine compounds using vibrational chiroptical spectroscopy. J Org Chem 2012; 77: 858-869.

6 Haesler J, Schindelholz I, Riguet E, Bochet CG, Hug W. Absolute configuration of chirally deuterated neopentane. Nature 2007; 446: 526-529.

7 Barron LD, Hecht L, McColl IH, Blanch EW. Raman optical activity comes of age. Mol Phys 2004; 102: 731-744.

8 Nafie LA. Vibrational optical activity in focus. App/ Spectrosc 1996; 50: A12.

9 Pecul M. New applications and challenges for computational ROA spectroscopy. Chirality 2009; 21: E98-E104.

10 Efrima S. The effect of large electric field gradients on the Raman optical activity of molecules adsorbed on metal surfaces. Chem Phys Lett 1983; 102: 79-82.

11 Efrima S. Raman optical activity of molecules adsorbed on metal surfaces: theory. J Chem Phys 1985; 83: 1356.

12 Etchegoin PG, Galloway C, Le Ru EC. Polarization-dependent effects in surfaceenhanced Raman scattering (SERS). Phys Chem Chem Phys 2006; 8: 2624-2648.

13 Janesko BG, Scuseria GE. Surface enhanced Raman optical activity of molecules on orientationally averaged substrates: theory of electromagnetic effects. J Chem Phys 2006; 125: 124704. 
14 Kneipp H, Kneipp J, Kneipp K. Surface-enhanced Raman optical activity on adenine in silver colloidal solution. Anal Chem 2006; 78: 1363-1366.

15 Abdalia S, Blanch EW. Surface enhanced Raman optical activity (SEROA). Chem Soc Rev 2008; 37: 980-992.

16 Yang N, Tang Y, Cohen AE. Spectroscopy in sculpted fields. Nano Today 2009; 4 269-279.

17 Novak V, Sebestík J, Bour P. theoretical modeling of the surface-enhanced Raman optical activity. J Chem Theory Comput 2012; 8: 1714-1720.

18 Dickson RM, Lyon LA. Unidirectional plasmon propagation in metallic nanowires. $J$ Phys Chem B 2000; 104: 6095-6098.

19 Fang Y, Wei H, Hao F, Nordlander P, Xu HX. Remote-excitation surface-enhanced Raman scattering using propagating Ag nanowire plasmons. Nano Lett 2009; 9: 2049-2053.

20 Huang Y, Fang Y, Sun MT. Remote excitation of surface-enhanced Raman scattering on single Au nanowire with quasi-spherical termini. J Phys Chem C2011; 115: 35583561 .

21 Lal S, Hafner JH, Halas NJ, Link S, Nordlander P. Noble metal nanowires: from plasmon waveguides to passive and active devices. Acc Chem Res 2012; 45: 1887-1895.

22 Helgert C, Pshenay-Severin E, Falkner M, Menzel C, Rockstuhl C et al. Chiral metamaterial composed of three-dimensional plasmonic nanostructures. Nano Lett 2011; 11: 4400-4404.

23 Schaferling M, Dregely D, Hentschel M, Giessen H. Tailoring enhanced optical chirality: design principles for chiral plasmonic nanostructures. Phys Rev X 2012; 2: 031010 .
24 Hug W, Hangartner G. A novel high-throughput Raman spectrometer for polarization difference measurements. J Raman Spectrosc 1999; 30: 841-852.

25 Sun Y, Xia YN. Large-scale synthesis of uniform silver nanowires through a soft, selfseeding, polyol process. Adv Mater 2002; 14: 833-837.

26 Sun M, Hou Y, Li Z, Liu L, Xu H. Remote excitation polarization-dependent surface photochemical reaction by plasmonic waveguide. Plasmonics 2011; 6: 681-687.

27 Chandrasekhar N, Mohiddon M, Chandrasekar R. Organic submicro tubular optical waveguides: self-assembly, diverse geometries, efficiency, and remote sensing properties. Adv Opt Mater 2013; 1: 305-311.

28 Frisch MJ, Trucks GW, Schlegel HB, Scuseria GE, Robb MA et al. Gaussian 09, Revision A.02. Wallingford, CT: Gaussian, Inc.; 2009.

29 Hohenberg P, Kohn W. Inhomogeneous electron gas. Phys Rev 1964; 136: B864B874.

30 Lee C, Yang W, Parr RG. Development of the Colle-Salvetti correlation energy formula into a functional of the electron density. Phys Rev B 1988; 37: 785-789.

31 Fang YR, Li ZP, Huang YZ, Zhang SP, Nordlander P et al. Branched silver nanowires as controllable plasmon routers. Nano Lett 2010; 10: 1950-1954.

32 Zhang S, Wei H, Bao K, Hakanson U, Halas NJ et al. Chiral surface plasmon polaritons on metallic nanowires. Phys Rev Lett 2011; 107: 096801.

cc) (i) (5)

This work is licensed under a Creative Commons Attribution-

NonCommercial-ShareAlike 3.0 Unported license. To view a copy of this license, visit http://creativecommons.org/licenses/by-nc-sa/3.0

Supplementary Information for this article can be found on Light: Science \& Applications' website (http://www.nature.com/lsa/). 